\title{
Validation and Explanation of Waterhammer-Based Locomotion
}

\author{
Ross L. Feller, Douglas P. Perrin, and Robert D. Howe \\ Harvard University \\ Division of Engineering and Applied Sciences \\ Cambridge, Massachusetts \\ feller@fas.harvard.edu; (dperrin, howe)@deas.harvard.edu
}

\section{ABSTRACT}

Search and rescue robots often use tethers to provide power and communication, but tethers get caught on debris and small robots have difficulty with the added drag of the tether. This work investigates a self-actuating tether capable of remaining free while traversing obstacles. We present a physical model of the pressure transients actuated tether. The model relates forward motion of the tether (independent of a dragging force) to the relevant design parameters of hose stiffness, flow diameter, tether length, applied pressure, and valve selection. We present an experiment to test and validate our model. The experimental results correspond within $15 \%$ to the expected values from our analysis and also validate the functional dependence of our model on the design parameters.

\section{INTRODUCTION}

Mobile robots are used to aid humans in environments that are too hazardous or inaccessible. This is especially true in post-disaster search-and-rescue sites where victims might be buried beneath unstable pileup. Rescue robots can make rescuers' jobs safer and easier by extending their reach in such severe conditions. After the World Trade Center (WTC) disaster on September 11, 2001, the limitations of rescue robots in such a chaotic environment became clear. The Center for Robotic Assisted Search and Rescue (CRASAR) deployed radio-controlled and tethered rescue robots eight times into the debris. CRASAR technicians named radio transmission failure [3] [4], poor maneuverability [2], and tether locking [3] as three of the most restrictive limitations they encountered.

Untethered, tele-operated robots were among the models used by CRASAR at the WTC site. Temporary loss of communication with these robots presented a problem for CRASAR technicians. Thick pieces of concrete and steel debris obstructing radio communication between robot and operator [4] were the likely cause of failure. In general tethered robots do not require batteries and telecommunications equipment. Therefore they can be built small, enabling them to penetrate further than their tethered counterparts into constricted search areas. The cost of using tethers is increase drag and a tendency to catch on obstacles, limiting the reach and path of the robot.

In [8] we investigated a novel solution to the problem of tether management, allowing search-and-rescue teams to take advantage of the benefits of tethering robots without the added obstruction to exploration. We proposed a solution involving flowing water through a hose within the tether and repeatedly closing a valve mounted on the robot. When the valve is closed the fluid rapidly decelerates, transferring energy to the valve and hose-an effect known as waterhammer. This energy transfer induces a forward motion of the tether and allows forward motion of the robot. Experiments with initial prototypes showed the solution works-allowing the robot to move forward in conditions where it would otherwise be stuck.

While the waterhammer phenomenon is well studied [5], the underlying mechanics of the tether motion in a flexible hose is unclear. The goal of this work is to relate the tether's performance (forward acceleration of the tether) to the relevant design parameters (e.g. tube material, flow diameter, tether length, applied pressure, valve selection). We begin our analysis by exploring the fundamental connection between the waterhammer and the motion of the tether. We then present an initial experiment that focuses our analysis on the dynamic interaction between the waterhammer wave and the flexible tube. Next, we develop a set of equations that relate the forward motion of the tether to the design parameters. Finally, we present experimental data for four different tethers to verify our mathematical model.

\section{INITIAL HyPOTHESIS}

A waterhammer-actuated tether is a complex system involving a coupled fluid-solid interaction. When a flow is rapidly arrested, the momentum of the fluid that is in motion is transferred to the parts of the system that are at rest (e.g. the valve, the tube, and the fluid that is already at rest). The momentum transfer from the decelerating fluid to the fluid that is at rest compresses the fluid and causes a substantial increase in pressure. The increase in pressure causes stress and deformation of the elastic tube.

We hypothesize that the waterhammer wave interacts with the wall of the elastic tube to directly accelerate the tether. Therefore tether motion would not be due only to a dragging force.

\section{OBSERVATIONS ON PINNING}

To test this we conducted an experiment where the valve was clamped at a fixed location (Fig. 1). We then placed an external mass on the tether. The mass was constrained only 


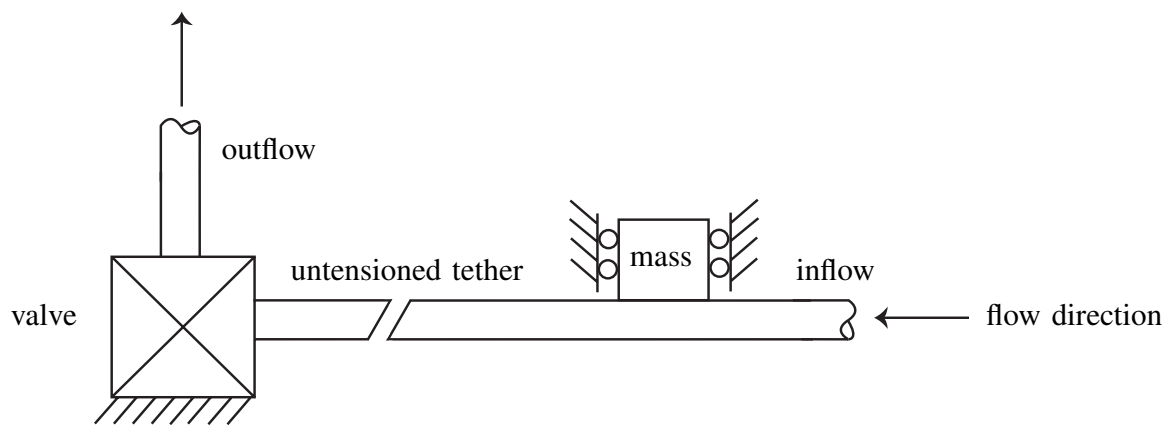

Fig. 1. Schematic of Experimental Setup

to move in the vertical direction with no friction. When the valve was actuated, the tether moved forward under the mass. We therefore can focus on this dynamic interaction between the waterhammer pressure wave and the elastic tether as an isolated (although possibly not the only) mechanism for tether motion.

\section{Physical Model}

We now present an analysis of the waterhammer-actuated tether to relate the performance of the system to the salient design parameters. We approach this problem by separating the analysis of the solid mechanics and the fluid mechanics. Throughout the analysis we assume that the tube is thin-walled and perfectly elastic. We also neglect the shear stress on the internal surface of the tube, i.e. we assume the viscosity of the fluid is negligible. First, we apply Newton's Law to the tube to relate the motion of the tube to the internal pressure. Then we analyze the fluid dynamics to relate the internal pressure to the design parameters.

\section{A. Momentum Balance}

We start with a free-body diagram of the small length of tube shown in Fig. 2, where $\sigma_{x}$ is the axial stress in the tube wall, $R$ is the radius of the tube, $T$ is the tube wall thickness assuming $T<<R, f_{r}$ is the frictional force per unit length, $t$ is time, and positive $x$ is taken in the direction of fluid flow. We are considering an acceleration in the positive $x$ direction. The momentum balance on the differential element is

$$
2 \pi R T \cdot\left[\sigma_{x}(x+d x)-\sigma_{x}(x)\right]-f_{r} \cdot d x=m \cdot d x \cdot \frac{\partial^{2} x}{\partial t^{2}}
$$

Dividing by $d x$ and noting the definition of $\frac{\partial}{\partial x}$ we have

$$
2 \pi R T \cdot \frac{\partial \sigma_{x}(x)}{\partial x}-f_{r}=m \cdot \frac{\partial^{2} x}{\partial t^{2}}
$$

This equation reveals that, if the force from the gradient of the axial stress is greater than the frictional force, the element of the tube will accelerate in the positive $x$ direction. This relates the performance of the tether (forward acceleration and motion) to the axial stress. We now need to relate the axial stress to the design parameters.

Following the classic analysis for a thin-walled cylindrical pressure vessel, we consider the momentum balance on the face of the tube in Fig. 3. Equating the axial stress $\sigma_{x}$ in the

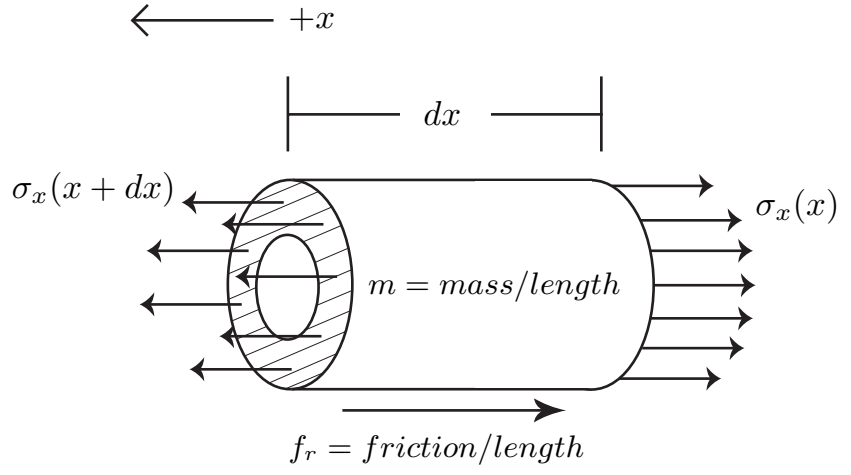

Fig. 2. Forces on a small element of tube

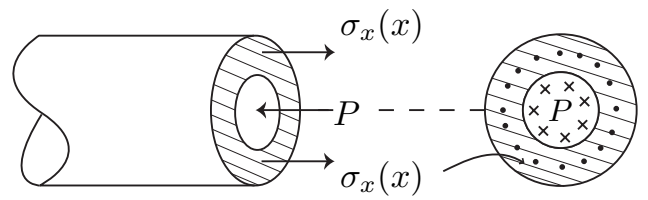

Fig. 3. Forces on cross-section of tube

tube wall and the pressure on the internal cross-section $P$, and solving for the axial stress, we find

$$
\begin{gathered}
2 \pi R T \cdot \sigma_{x}=\pi R^{2} P \\
\sigma_{x}=\frac{R P}{2 T}
\end{gathered}
$$

If we assume the tube is purely elastic, i.e. assume the tube responds immediately to a given load, then Eqn. 4 is valid if both the axial stress and internal pressure are dependent on time and space

$$
\sigma_{x}(x, t)=\frac{R P(x, t)}{2 T}
$$

Substituting Eqn. 5 into Eqn. 2, we relate the acceleration to the internal pressure

$$
\pi R^{2} \cdot \frac{\partial P(x, t)}{\partial x}-f_{r}=m \cdot \frac{\partial^{2} x}{\partial t^{2}}
$$

\section{B. Waterhammer Theory}

To relate the internal pressure to the design parameters, we need to investigate the pressure waves induced by a waterhammer. We will first examine the waterhammer effect by inspecting the Navier-Stokes equations and then with a closer look at a small element of fluid. 
To determine the equation for the velocity of a waterhammer wave, it is necessary to consider a small element of fluid in the pipe. The fluid is assumed to be elastic: between time $t$ and time $t+\delta t$ the element has compressed in length and expanded in cross section and has not necessarily maintained constant volume. Considering the change in volume of the tube due to deformation and the change in volume of the fluid due to the compressibility of water, the conditions of dynamic equilibrium and continuity yield the fundamental waterhammer equations, relating fluid pressure to flow velocity (from [7])

$$
\begin{gathered}
\Delta P_{W H}=-\rho c_{\text {wave }} \Delta u \\
\frac{\partial P_{W H}}{\partial x}=-\rho \frac{\partial u}{\partial t} \\
\frac{\partial P_{W H}}{\partial t}=-c_{\text {wave }}^{2} \rho \frac{\partial u}{\partial x} \\
c_{\text {wave }}=\sqrt{\frac{K}{\rho}}\left(1+\frac{D s K}{E T}\right)^{-\frac{1}{2}}
\end{gathered}
$$

where $c_{\text {wave }}$ is the wave speed, $K$ is the bulk modulus of the fluid, $D, E$, and $T$ are the diameter, Young's modulus, and thickness of the tube, and $s$ is a constant determined by the constraints on the deformation of the pipe in a longitudinal direction (if the pipe is free to expand, $s=\frac{5}{4}-\nu$, where $\nu$ is Poisson's ratio for the pipe material). Eqns. 8 and 9 can be solved simultaneously to give

$$
\begin{gathered}
P_{W H}-P_{o}=f\left(t-\frac{x}{c_{\text {wave }}}\right)+F\left(t+\frac{x}{c_{\text {wave }}}\right) \\
u-u_{o}=\frac{1}{\rho c_{\text {wave }}}\left[f\left(t-\frac{x}{c_{\text {wave }}}\right)-F\left(t+\frac{x}{c_{\text {wave }}}\right)\right]
\end{gathered}
$$

Here $f$ describes a pressure wave propagating in the positive $x$ direction (the flow direction) and $F$ describes a wave propagating in the negative $x$ direction (opposite the flow direction). Therefore at any time $t$, the pressure at any position $x$ is given by the sum of the two waves propagating in opposite directions. Because the waterhammer equations still depend on the fluid velocity flowing through the tube, we now consider the relation between the flow velocity and the design parameters.

\section{Macroscopic Mechanical Energy Balance}

If we consider a system with one entrance and one exit, the general form of this macroscopic mechanical energy balance is

$$
\left.\left(\frac{P}{\rho g}+\frac{\alpha\langle u\rangle^{2}}{2 g}+z\right)\right|_{\text {entrance }} ^{\text {exit }}=h_{\text {pump }}-h_{\text {losses }}
$$

where $P$ is the pressure, $\rho$ is the fluid density, $g$ is the acceleration due to gravity, $\langle u\rangle$ is the average velocity over a given cross section, $z$ is the vertical height, $h_{\text {pump }}$ is the pump head, $h_{\text {losses }}$ is the head loss due to viscous losses and restrictions, and $\alpha$ is a constant determined by the velocity profile (for fully developed turbulent flow $\alpha \approx 1$ ). This equation (the engineering Bernoulli equation) represents the conservation of mechanical energy for a general piping system.
In our situation we assume that there is no change in velocity or height between the entrance and the exit and that $h_{\text {pump }}=0$. The energy balance then reduces to:

$$
\left.\left(\frac{P}{\rho g}\right)\right|_{\text {exit }} ^{\text {entrance }}=h_{\text {losses }}
$$

where $h_{\text {losses }}=h_{\text {tube }}+h_{\text {valve }}$. The viscous loss due to the valve is expressed as:

$$
h_{\text {valve }}=\frac{1}{2} u^{2} k_{\text {valve }}
$$

where $k_{\text {valve }}$ is the friction loss factor for the valve. It is necessary to relate the friction loss factor for the valve (as defined in Eqn. 15) to the relevant design specification for solenoid valves. The viscous loss for solenoid valves is typically given as the coefficient of volume, $C_{v}$, relating the volume flow rate through the valve to the pressure differential across the valve:

$$
\text { Volume flow rate }=C_{v} \sqrt{\frac{\Delta P}{\text { specific gravity of fluid }}}
$$

To solve for the friction loss factor, we relate the volume flow rate to the linear flow rate and note the specific gravity of water is 1 :

$$
u \pi R^{2}=C_{v} \sqrt{\Delta P_{\text {applied }}}
$$

and

$$
u^{2}=\frac{C_{v}^{2} \Delta P_{\text {applied }}}{\pi^{2} R^{4}}
$$

Considering the valve as the entire system, the energy balance (Eqn. 14) gives:

$$
\begin{gathered}
\frac{\Delta P_{\text {applied }}}{\rho g}=h_{\text {valve }}=\frac{1}{2} u^{2} k_{\text {valve }} \\
\frac{\Delta P_{\text {applied }}}{\rho g}=\frac{1}{2} \frac{C_{v}^{2} \Delta P_{\text {applied }}}{\pi^{2} R^{4}} k_{\text {valve }}
\end{gathered}
$$

and solving for $k_{\text {valve }}$ :

$$
k_{v a l v e}=\frac{2 \pi^{2} R^{4}}{\rho g C_{v}^{2}}
$$

We can express the viscous losses in the tube, $h_{t u b e}$, using the Moody friction factor[6], $f_{m}$, as

$$
h_{\text {tube }}=\frac{1}{2} f_{m} \frac{L}{D} \frac{u^{2}}{g}
$$

Given the Reynolds number $\mathcal{R}=\frac{u D}{\nu_{v i s c}}$ (where $\nu_{\text {visc }}=\frac{\mu}{\rho}$ is the dynamic viscosity), and assuming a hydraulically smooth tube, the Blasius approximation for the friction factor ([1] valid for $2.1 \times 10^{3}<\mathcal{R}<10^{5}$ ) is:

$$
f_{m}=\frac{0.3164}{\mathcal{R}^{\frac{1}{4}}}=\frac{0.3164 \nu_{v i s c}^{\frac{1}{4}}}{u^{\frac{1}{4}} D^{\frac{1}{4}}}
$$

Substituting into Eqn. 16, we express $h_{t u b e}$ as

$$
h_{\text {tube }}=\frac{0.1582 \nu_{v i s c}^{\frac{1}{4}} L}{g D^{\frac{5}{4}}} u^{\frac{7}{4}}
$$


We can now write the energy balance for the system as:

$$
\begin{gathered}
\frac{\Delta P_{\text {applied }}}{\rho g}=\frac{1}{2} f_{m} \frac{L}{D} \frac{u^{2}}{g}+\frac{1}{2} k_{\text {valve }} u^{2} \\
\frac{\Delta P_{\text {applied }}}{\rho}=\frac{0.1582 \nu_{v i s c}^{\frac{1}{4}} L}{D^{\frac{5}{4}}} u^{\frac{7}{4}}+\frac{\pi^{2} R^{4}}{\rho C_{v}^{2}} u^{2}
\end{gathered}
$$

This final equation relates the flow velocity to the relevant design parameters (flow diameter $D$ and radius $R$, tether length $L$, applied pressure $\Delta P_{\text {applied }}$, and the valve's coefficient of volume $C_{v}$ ).

\section{Summary}

The following equations derived above relate the forward acceleration of a waterhammer-actuated tether to the relevant design parameters (flow diameter, tether material, tether length, applied pressure, and valve specifications).

Momentum Balance dynamic force balance on a differential element of the tether relates acceleration to change in pressure

$$
\pi R^{2} \cdot \frac{\partial P(x, t)}{\partial x}-f_{r}=m \cdot \frac{\partial^{2} x}{\partial t^{2}}
$$

Waterhammer Equations relates the fluid pressure to flow velocity

$$
\begin{gathered}
\Delta P_{W H}=-\rho c_{\text {wave }} \Delta u \\
c_{\text {wave }}=\sqrt{\frac{K}{\rho}}\left(1+\frac{D s K}{E t}\right)^{-\frac{1}{2}}
\end{gathered}
$$

Engineering Bernoulli Equation macroscopic mechanical energy balance on the tether system relates velocity to tether parameters

$$
\frac{\Delta P_{\text {applied }}}{\rho}=\frac{0.1582 \nu_{v i s c}^{\frac{1}{4}} L}{D^{\frac{5}{4}}} u^{\frac{7}{4}}+\frac{\pi^{2} R^{4}}{\rho C_{v}^{2}} u^{2}
$$

\section{Methods and Materials}

To verify the preceding theoretical analysis, we instrumented an actuated tether system with force and pressure sensors. All experiments used a total inflow length of $3 \mathrm{~m}$ $(1 \mathrm{~m}$ between the valve and force sensor and $2 \mathrm{~m}$ between the force sensor and source) and an outflow length of $3 \mathrm{~m}$. We selected four tubes, varying material stiffness, flow diameter, and wall thickness across trials (Table I). The tubes were made of either linear low-density polyethylene (LLDPE) or semirigid polypropylene. The typical range of Young's modulus for each material is listed in Table I; for both materials we assume a Poisson's ratio of 0.4. Water was supplied from a 20 gallon holding tank (Model ELSF-20, A.O.Smith, max. working pressure: $150 \mathrm{psi}$ ) pressurized with compressed air at 70 psi. All experiments used a direct-acting normallyopen solenoid valve (model 71295SN2KNJ1, Parker Valves) to control the fluid flow.

We measured the linear flow velocity by noting the time to fill a $10 \mathrm{~L}$ container and dividing the volume flow rate by the cross-sectional area. We measured the force in the axial direction and pressure in the line using a custom mounting plate (Fig. 4) attached a force sensor with a resolution of $0.025 \mathrm{~N}$ (Gamma model, ATI Industrial Automation Inc). The
TABLE I

TUBES USED IN EXPERIMENTS

\begin{tabular}{cccccc} 
Tube & Material & $\begin{array}{c}\text { Modulus } \\
(\mathrm{MPa})\end{array}$ & $\begin{array}{c}\text { ID } \\
\text { (in) }\end{array}$ & $\begin{array}{c}\text { OD } \\
\text { (in) }\end{array}$ & $\begin{array}{c}\text { Wall } \\
\text { Thickness (in) }\end{array}$ \\
\hline 1 & LLDPE & $200-500$ & $\frac{1}{8}$ & $\frac{1}{4}$ & $\frac{1}{16}$ \\
2 & LLDPE & $200-500$ & $\frac{1}{8}$ & $\frac{3}{8}$ & $\frac{1}{8}$ \\
3 & LLDPE & $200-500$ & $\frac{1}{4}$ & $\frac{3}{8}$ & $\frac{1}{16}$ \\
4 & Polypropylene & $700-1200$ & $\frac{1}{4}$ & $\frac{3}{8}$ & $\frac{1}{16}$ \\
\multicolumn{5}{c}{ TABLE II } \\
\multicolumn{5}{c}{ FLOW VELOCITY } \\
Tube & Theoretical & Measured & Percent & Reynolds \\
\multicolumn{7}{c}{ Velocity $(\mathrm{m} / s)$} & Velocity $(\mathrm{m} / s)$ & Error & Number \\
\hline 1 & 3.81 & 4.13 & $8.4 \%$ & 13,100 \\
2 & 3.81 & 4.28 & $12.3 \%$ & 13,600 \\
3 & 2.31 & 2.59 & $12.1 \%$ & 16,400 \\
4 & 2.31 & 2.57 & $11.2 \%$ & 16,300
\end{tabular}

fluid pressure was measured with a silicon pressure transducer (Model ST250PG1SPRF, Honeywell Sensing, resolution = $2.5 \mathrm{psi}$ ) capable of sensing changes of $225 \mathrm{psi}$ in less than $500 \mu \mathrm{s}$. We recorded the force and pressure data using a data acquisition board at a sampling rate of $1000 \mathrm{~Hz}$ with a resolution of 3.2 bits per volt. We recorded three trials for both valve closing and valve opening for each tube material. In addition, we measured the frictional dead band (when an applied force less than the static frictional force results in no motion) for the four tubes for a pinning mass of $670 \mathrm{~g}$.

\section{RESULTS}

\section{A. Flow Velocity}

The measured flow velocity for each tube is given in Table II. We predicted the theoretical velocity using Eqn. 21, where the percent error is given in terms of deviation from the theoretical flow. All flow velocities are for a $3 \mathrm{~m}$ inflow and $3 \mathrm{~m}$ outflow hose. The Reynolds numbers for the measured flows are within the range $\left(2.1 \times 10^{3}<(R)<10^{5}\right)$, validating our use of the Blasius approximation for the friction factor. The measured flow velocities are approximately $10 \%$ above the theoretical prediction, however the ratio of flow velocities between the two different cross-sections correspond well to the theoretical ratio, producing a measured decrease in velocity of $38.6 \%$ versus an expected decrease of $39.4 \%$. In our approximation of the friction factor, we assume a hydraulically smooth tube. Using this assumption, we would expect to have slower velocities than the prediction, not faster. The error in our prediction is more likely due to an error or variability in the coefficient of volume specification for the valve. Despite this error, Eqn. 21 is still useful for tether design, providing an accurate prediction for how flow velocity changes as the result of increasing or decreasing different tether parameters. In our experiment, the flow velocities decrease with the increase in cross-sectional area between Tubes \#1 and \#2 and Tubes \#3 and \#4.

\section{B. Force-Pressure Plots}

The force in the direction of the valve and pressure plots for valve closing for Tube \#2 are shown in Fig. 5. The valve was 


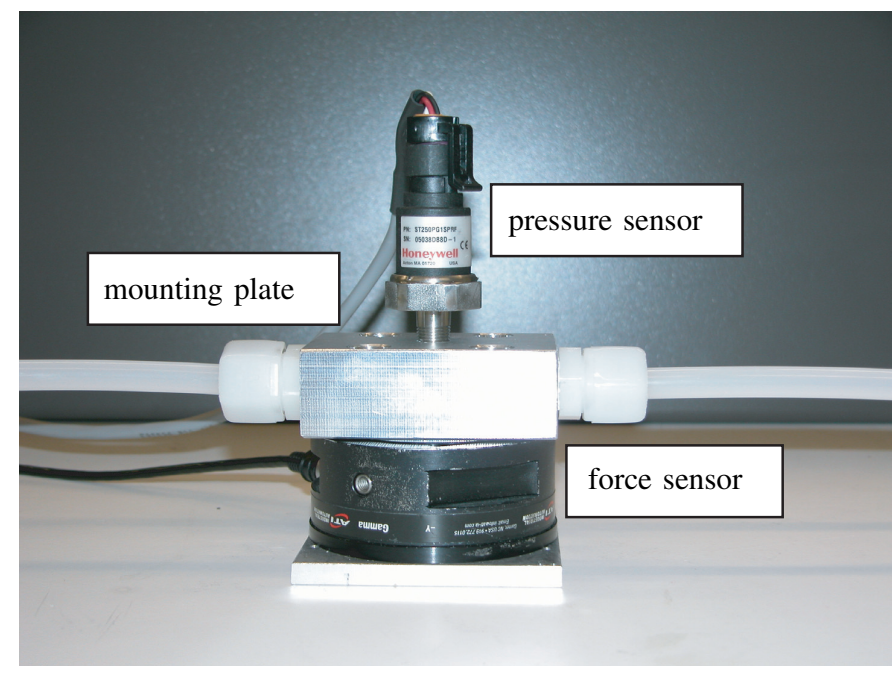

Fig. 4. Force and Pressure Sensors with Mounting Plate
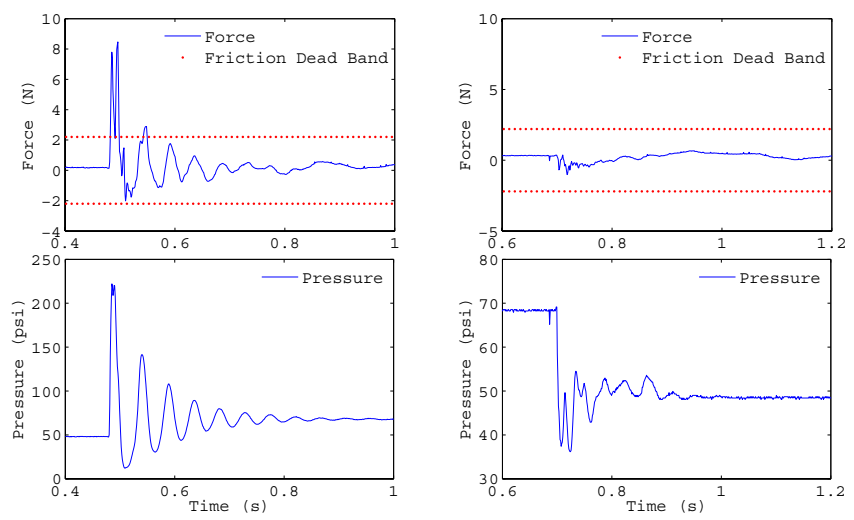

Fig. 5. Force and Pressure Plots for Valve Closing (left) and Opening (right) for Tube \#2

initially open with a fully developed flow, the valve was then closed for the remainder of the trial. Pressure was measured halfway between the reservoir and the valve (Fig. 4). Forward motion of the tether is dependent on a number of variables. In one example with a 670 gram pining mass the tether moved $4.5 \mathrm{~mm}$ toward the valve with each valve closure (pulse).

When the valve is closed a positive pressure wave propagates from the valve.This wave bounces between the reservoir and the valve until the pressure everywhere in the tube is equal to the pressure in the reservoir. The reflection of these waves is evident from the oscillation of the pressure signals in the plots in Fig. 5.

The force plots show an initial increase in the force in the direction of the valve, then an oscillation and decay similar to the pressure signals. The frictional dead band is shown on the same plots for a pinning mass of $670 \mathrm{~g}$. The force must exceed the dead band in order to cause motion.

The valve was initially closed with no flow, then the valve was opened for the remainder of the trial. When the valve is opened, flow develops across the valve and a negative pressure wave propagates opposite the direction of flow. The magnitude of the pressure wave and the force for valve opening is less
TABLE III

Magnitude of Pressure Wave for Valve Closing

\begin{tabular}{cccc} 
Tube & $\begin{array}{c}\text { Measured } \\
\mathbf{\Delta} \mathbf{P}_{\max }(\mathbf{p s i})\end{array}$ & $\begin{array}{c}\text { Calculated } \\
\boldsymbol{\Delta} \mathbf{P}_{\max }(\mathbf{p s i})\end{array}$ & $\begin{array}{c}\text { Theoretical } \\
\mathbf{\Delta} \mathbf{P}_{\max }(\mathbf{p s i})\end{array}$ \\
\hline 1 & 159.3 & 132.7 & $151.6-233.8$ \\
2 & 186.8 & 158.6 & $184.2-280.7$ \\
3 & 96.3 & 105.0 & $71.9-111.7$ \\
4 & 166.9 & 149.5 & $131.1-184.0$
\end{tabular}

than for valve closing. For valve closing, there is a fully developed flow - when the valve is closed the flow velocity goes to zero in the time it takes the solenoid to actuate. For valve opening, the flow develops as the valve opens. The combination of the valve opening slower than closing, and the added time to develop a flow for valve opening account for the reduced magnitude of the waterhammer wave.

\section{Magnitude of Pressure Wave}

Table III compares the maximum magnitudes of the measured pressure to the expected pressure magnitudes. The measured magnitude is the average pressure change for the first pressure peak across the three trials for each tube. The calculated magnitude is computed from Eqn. 19 using the measured values for flow rate (Table II) and wave speed (from Fourier analysis of the pressure signal). The theoretical magnitude is calculated using the theoretical values for flow rate and wave speed from Eqns. 20 and 21. The measured experimental results correspond reasonably well to the calculated magnitudes (within 20\%) and fall within the range of theoretical values.

\section{Magnitude of Force Wave}

For all four tubes, the initial force peak is greater than the friction dead band, resulting in forward motion for pinning mass of $670 \mathrm{~g}$. The initial peak is above the dead band for $15-20 \mathrm{~ms}$, the same amount of time that we observed forward motion of the tether using high-speed video. From Eqn. 18, the force resulting from the waterhammer wave is:

$$
F_{\text {waterhammer }}\left(x=x^{*}, t\right)=\left.\pi R^{2} \cdot \frac{\partial P(x, t)}{\partial x}\right|_{x^{*}}
$$

We estimate the pressure gradient as:

$$
\left.\frac{\partial P(x, t)}{\partial x}\right|_{x^{*}} \approx \frac{\Delta P}{\Delta x}=\frac{\Delta P}{\Delta t} \frac{\Delta t}{\Delta x}=\frac{\Delta P}{\Delta t} \frac{1}{c_{\text {wave }}}
$$

where $\Delta P$ is the measured $\Delta P_{\max }$ from Table III, $\Delta t$ is the rise time corresponding to $\Delta P_{\max }$, and $c_{\text {wave }}$ is the measured wave speed. The average values calculated from Eqn. 22 for the four tubes are shown in Table IV, along with the measured experimental maximum force for each tube. The force peak increases substantially when we increase cross-sectional area, despite a smaller $\Delta P_{\max }$ for Tube \#3 and Tube \#4. This observed dependence and the correlation of measured and expected values in Table IV validate using Eqn. 18 in tether design. 
TABLE IV

MAgnitude of Force FOR VALVE Closing

\begin{tabular}{ccc} 
Tube & $\begin{array}{c}\text { Measured } \boldsymbol{\Delta} \mathbf{F}_{\text {max }} \\
(\mathrm{N})\end{array}$ & $\begin{array}{c}\text { Calculated } \boldsymbol{\Delta} \mathbf{F}_{\max } \\
(\mathrm{N})\end{array}$ \\
\hline 1 & 6.98 & 7.39 \\
2 & 8.19 & 7.81 \\
3 & 16.15 & 13.17 \\
4 & 23.30 & 22.66
\end{tabular}

\section{DISCUSSION}

This paper presents a theoretical framework to explain part of the mechanism used for actuation waterhammer-driven tethers. Our goal was to investigate a waterhammer actuated tether system for rescue robots in order to determine the fundamental relationship between the performance of the tether system and the salient design parameters. Optimizing the design parameters in Eqns. 18-21 is not a trivial task. The equations are non-linear and interrelated. However we can provide an initial intuition for design based on these four equations.

Valve Properties: It is important to have a fast-closing valve to maximize the change in velocity of the fluid and the magnitude of the waterhammer wave. In order to prevent motion in the reverse direction, depending on the frictional forces encountered and the pulling capabilities of a given robot, it may be important to have a valve that opens slowly. In our experiments, the frictional dead band provided a motion bias in the forward direction without a force bias on the valve. In a realistic environment, the forward force provided by the robot and the frictional forces would both bias the motion of the tether. A normally-open solenoid valve closes when the solenoid is actuated, creating a magnetic force to close the orifice. When the solenoid is not powered, a spring acts to open the orifice.

It is also important for the valve to have a large coefficient of volume. A larger coefficient of volume increases the volume flow rate for a given pressure differential, increasing the linear flow rate and the magnitude of the waterhammer wave.

Hose Diameter: Increasing the cross-sectional area of the hose will increase the force due to the waterhammer wave and therefore increase the performance of the system. Increasing the diameter will also cause a decrease in the waterhammer wave speed, although this is a weak relation and was not verified by the experimental results. Increasing the flow diameter decreases the viscous loss in the tube, allowing for a faster flow rate. However, increasing the flow diameter increases the viscous loss in the valve. The valve can only handle a given volume flow rate for a given pressure differential. Increasing the flow diameter will reduce the viscous loss in the tube, increasing the pressure differential across the valve. However, this is not sufficient to accommodate the increase in volume flow rate and therefore the linear flow velocity will decrease. The decrease in flow velocity will decrease the magnitude of the waterhammer pressure wave. It is necessary to choose a flow diameter that maximizes the force due to the waterhammer wave without decreasing the flow velocity to the point where the decreased magnitude of the pressure wave cancels out the increase in cross-sectional area.

Hose Thickness: Hose thickness does not have a strong effect on the performance of the tether system. As our experiment shows, an increase in hose thickness will cause a slight increase in waterhammer wave speed. However, it is important to have a hose thick enough to withstand the pressures associated with a given system configuration, and it may be beneficial to embed power and communications wires in the tube wall.

Applied Pressure: Increasing the applied pressure will increase the linear flow velocity therefore increasing the magnitude of the waterhammer wave. However, the pressure ratings on the valve and hose limit the maximum applied pressure.

Tether Length: Increasing the length of the tether will increase the viscous losses in the tube, decreasing the flow rate and the magnitude of the waterhammer wave.

Tube Material: A stiffer hose material will increase the waterhammer wave velocity and therefore increase the magnitude of the waterhammer pressure wave. A stiffer hose will also decrease the decay of the waterhammer wave, as seen in Fig. 5. Depending on the tension provided by the robot and the frictional forces, this effect may cause motion in both the forward and reverse directions. However, the motion in the reverse direction can be eliminated by arresting the waterhammer wave at the reservoir.

\section{AcKnowledgments}

The authors would like to thank the Center for RobotAssisted Search and Rescue (CRASAR) for a wealth of information about search and rescue robots, tethers, and real disaster sites. We would also like thank Albert Kwon, Prof. Howard Stone, Amy Kerdok, and Prof. John Hutchinson for their contributions to this work.

\section{REFERENCES}

[1] R. Bird, W. Stewart, and E. Lightfoot. Transport Phenomena. John Wiley and Sons, Inc., New York, NY, 1960.

[2] M. R. Blackburn, H. R. Everett, and R. T. Laird. After action report to the joint program office: Center for robotic assisted search and rescue (CRASAR) related efforts at the World Trade Center. Technical Report TD 3141, Space and Naval Warfare Systems Command, Aug. 2002.

[3] J. Casper. Human-robot interactions during the robot-assisted urban search and rescue response at the World Trade Center. Master's thesis, Computer Science and Engineering, University of South Florida, Apr. 2002.

[4] A. Jacoff, E. Messina, and J. Evans. A standard test course for urban search and rescue robots. In Proceedings of the IEEE Performance Metrics for Intelligent Systems Workshop, Gaithersburg, MD, Aug. 2000.

[5] L. W. Mays and Y.-K. Tung. Hydrosystems Engineering and Management. McGraw-Hill, 1991.

[6] L. Moody. Friction factors for pipe flow. ASME Transactions, 66:671, 1944.

[7] J. Parmakian. Waterhammer Analysis. Dover Publications, New York, NY, 1963.

[8] D. Perrin, A. Kwon, and R. Howe. A novel actuated tether design for rescue robots using hydraulic transients. In Proceedings of the IEEE International Conference on Robotics and Automation, New Orleans, LA, USA, 2004. 